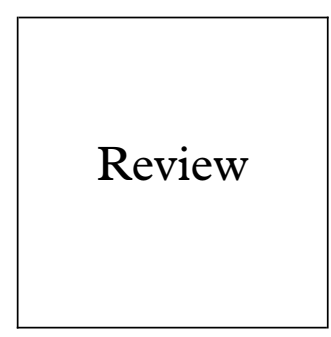

\title{
HIV drug resistance: genotypic assays and their possible applications
}

\author{
A L Ballard, P A Cane, D Pillay
}

\section{Introduction}

The replication of HIV, like all other retroviruses, is characterised by the conversion of RNA into complementary single stranded DNA and then double stranded DNA by the viral enzyme reverse transcriptase. This double stranded DNA integrates into the host chromosome to become "proviral" DNA. In turn the proviral DNA acts a template for the production of further viral genomic RNA and also viral messenger RNA. The messenger RNA is translated into viral proteins that together with the viral genomic RNA become assembled into new HIV virions. ${ }^{1}$

\section{Viral genetic variation}

HIV RNA, as for any RNA, comprises a polymer of ribonucleotides containing the bases adenine $(A)$, cytosine $(C)$, guanine $(G)$, and uracil (U). By comparison, DNA is composed of deoxyribonucleotides with identical bases except that thymidine (T) replaces uracil. Nucleic acid replication, catalysed by the appropriate polymerase, generates a complementary strand to the initial template, whereby a $\mathrm{G}$ base pairs with $\mathrm{C}$ on the complementary strand (or vice versa) and A pairs with $\mathrm{T}$ (in DNA) or U (in RNA). The HIV genome sequence should, therefore, be conserved during replication via the accurate conversion of RNA into proviral DNA, or proviral DNA back into genomic RNA or messenger RNA. It is the base sequence of the messenger RNA that determines the sequence of the amino acids in the viral proteins. Each amino acid is coded for by a specific triplet of bases (a codon) and since there are 64 possible triplet combinations and only 20 amino acids, most amino acids are encoded by more than one triplet.

Although the base pairing of $\mathrm{G}$ with $\mathrm{C}$ and $\mathrm{A}$

Antiviral Susceptibility Reference Unit, Public Health Laboratory,

Birmingham

Heartlands and

Solihull NHS Trust,

Heartlands Hospital,

Birmingham

A L Ballard

P A Cane

D Pillay

Correspondence to: Dr D Pillay, Antiviral Susceptibility Reference Unit, Public Health Laboratory, Heartland Hospital, Bordersley Green East, Birmingham B9 5SS.

Accepted for publication 12 June 1998 (nous) or result in amino acid char (non-synonymous). When amino acid substitutions do occur the resulting protein can have altered structure and function. It should be transcriptase is high, at $10^{-4} / \mathrm{b}$ ase - that is, one eracy mentioned above, these mistakes or "point mutations" can either be silent (synnucleic acid, all polymerases make base incorprrors. DNA dependent errors, thus achieving high fidelity replication. read and consequently the conversion of retroviral RNA into DNA is of relatively low fidelity. The estimated error rate for reverse base pair error for every 10000 bases noted that some mutations, such as the $G$ to $A$ mutation, are more liable to occur than others. None the less, assuming a random distribution of mutations at a rate of $10^{-4} /$ base with a daily production rate of $10^{8} \mathrm{HIV}$ virions, it is likely that each and every possible mutation in the HIV genome is produced many times each day in an infected individual. The predominant species at any one time will represent the most replication competent ("fittest") within the prevailing selective environment. Many variants will have reduced fitness, or indeed be defective for replication, and so will not survive. Even in an untreated patient, therefore, a diverse virus population exists ("quasispecies") which, although predominantly "wild type" in nature, will possibly include drug resistant variants as minor species.

\section{Antiretroviral drug therapy}

It is now widely accepted that the clinical benefit associated with potent antiretroviral therapy is mediated through a reduction in viral replication. Also, if viral replication can be suppressed to very low levels the opportunity for viral variation is reduced and the emergence and selection of drug resistant strains is much slower.

At present there are three major classes of drugs used in HIV treatment. These drugs are active against the viral reverse transcriptase and protease, both of which are essential enzymes for HIV replication. Nucleoside analogue reverse transcriptase inhibitors act by competing with the natural nucleosides for the active site of the enzyme and on incorporation into the extending DNA strand stop DNA synthesis (chain termination). Decreased virus susceptibility (that is, an increase in the $\mathrm{IC}_{50}$, the concentration of drug required to inhibit viral replication by $50 \%$ ) to this group of compounds is thought to arise by amino acid changes that alter the structure of the active site such that the mutant form preferentially binds the natural substrate.

Non-nucleoside reverse transcriptase inhibitors (NNRTIs) produce their inhibitory effect by binding to a hydrophobic pocket near the catalytic site. Drug resistance to NNRTIs arises through amino acid substitutions that change the binding of the drug to this hydrophobic pocket.

Protease inhibitors (PIs) are a potent group of antiretroviral drugs most of which mimic the peptide substrate and bind to the protease active site. Resistance is again generated as a result of amino acid substitutions at or near the active site that alter drug binding. The key mutations associated with resistance to the 
Table $1 \mathrm{~A}$ Key mutations in HIV-1 reverse transcriptase and protease genes associated with resistance to important antiretroviral drugs in clinical use (adapted from Schinazi et al ${ }^{3}$. Nucleoside reverse transcriptase inhibitors

\begin{tabular}{|c|c|c|}
\hline Antiviral drug & Key amino acid changes associated with resistance & Comments \\
\hline Zidovudine (AZT) & M41L, D67N, K70R, L210W, T215Y, K219Q & $\begin{array}{l}\text { No cross resistance to any other inhibitors. For high level } \\
\text { resistance( } 100 \text {-fold), at least three mutations, including } 41 \text { and } 215 \\
\text { required. When } 184 \text { mutation present (combination therapy) resistance to } \\
\text { AZT requires more than three mutations. }\end{array}$ \\
\hline Didanosine (ddI) & K65R, L74V, V75T, M184V & $\begin{array}{l}\text { Codon } 74 \text { mutation suppresses the effect of } 215 \text { AZT resistance mutation. } \\
\text { Cross resistance to ddC. }\end{array}$ \\
\hline Zalcitabine (ddC) & K65R, T69D, L74V, V75T, M184V & $\begin{array}{l}\text { Mutations conferring resistance to ddC rarely seen in clinical isolates and } \\
\text { high level resistance has not been observed. }\end{array}$ \\
\hline Lamivudine (3TC) & $\mathrm{M} 184 \mathrm{~V} / \mathrm{I} / \mathrm{T}$ & $\begin{array}{l}\text { Codon } M 184 \mathrm{~V} \text { mutation confers high level resistance }(1000-\text { fold), but the } \\
\text { virus has a decreased replication rate. } 184 \text { mutation suppresses the effect of } \\
\text { AZT } 215 \text { resistance mutation. In the majority of patients the } 184 \text { mutation } \\
\text { resensitises virus to zidovudine. }\end{array}$ \\
\hline Abacavir (1592) & K65R, L74V, Y115F, Q151M & $\begin{array}{l}\text { Preliminary data suggest that higher level phenotypic resistance is } \\
\text { associated with a greater number of mutations and this determines } \\
\text { virological response. }\end{array}$ \\
\hline Multiple nucleoside analogues & A62V, V75I, F77L, F116Y, Q151M & $\begin{array}{l}\text { Mutation at } 151 \text { alone confers multidrug resistance. Observed in a } \\
\text { significant proportion of individuals receiving long term therapy with two } \\
\text { or more drugs. }\end{array}$ \\
\hline
\end{tabular}

^Amino acids: $\mathrm{A}=$ alanine; $\mathrm{C}=$ cysteine; $\mathrm{D}=$ aspartic acid; $\mathrm{E}=$ glutamic acid; $\mathrm{F}=$ phenylalanine; $\mathrm{G}=$ glycine; $\mathrm{H}=$ histidine; $\mathrm{I}=$ isoleucine; $\mathrm{K}=$ lysine; $\mathrm{L}=$ leucine; $\mathrm{M}=$ methionine; $\mathrm{N}=$ asparagine; $\mathrm{P}=$ proline; $\mathrm{Q}=$ glutamine; $\mathrm{R}=$ arginine; $\mathrm{S}=$ serine; $\mathrm{T}=$ threonine; $\mathrm{V}=$ valine; $\mathrm{W}=$ tryptophan; $\mathrm{Y}=$ tyrosine.

Table $1 B$ Key mutations in HIV-1 reverse transcriptase and protease genes associated with resistance to important antiretroviral drugs in clinical use (adapted from Schinazi et al $)^{3}$. Non-nucleoside reverse transcriptase inhibitors

\begin{tabular}{lll}
\hline Antiviral drug & Key amino acid changes associated with resistance & Comments \\
\hline Nevirapine & K103N, V106A, V108I, Y181C, Y188C, G190A & $\begin{array}{l}\text { Codon 181 mutation confers 100-200-fold reduction in sensitivity and is } \\
\text { associated with cross resistance to other non-nucleoside RT inhibitors. } \\
\text { Codon 236 mutation confers high level resistance to delavirdine, but } \\
\text { increases susceptibility to nevirapine. Codon 236 mutation rarely seen in } \\
\text { clinical isolates probably as a result of reduced fitness. }\end{array}$ \\
Efavirenz & $\mathrm{K} 103 \mathrm{~N}, \mathrm{~L}$ (00) & Codon 103 change is most common in clinic, although 188 and 190 changes \\
confer greater phenotypic resistance. Limited in vivo data at present.
\end{tabular}

^Amino acids: $\mathrm{A}=$ alanine; $\mathrm{C}=$ cysteine; $\mathrm{D}=$ aspartic acid; $\mathrm{E}=$ glutamic acid; $\mathrm{F}=$ phenylalanine; $\mathrm{G}=$ glycine; $\mathrm{H}=$ histidine; $\mathrm{I}=$ isoleucine; $\mathrm{K}=$ lysine; $\mathrm{L}=$ leucine; $\mathrm{M}=$ methionine; $\mathrm{N}=$ asparagine; $\mathrm{P}=$ proline; $\mathrm{Q}=$ glutamine; $\mathrm{R}=$ arginine; $\mathrm{S}=$ serine; $\mathrm{T}=$ threonine; $\mathrm{V}=$ valine; $\mathrm{W}=$ tryptophan; $\mathrm{Y}=$ tyrosine.

Table $1 C$ Key mutations in HIV-1 reverse transcriptase and protease genes associated with resistance to important antiretroviral drugs in clinical use (adapted from Schinazi et al $^{3}$ ). Protease inhibitors

\begin{tabular}{|c|c|c|}
\hline Antiviral drug & Key amino acid changes associated with resistance* & Comments \\
\hline Ritonavir & $\mathrm{V} 82 \mathrm{~A} / \mathrm{F} / \mathrm{S} / \mathrm{T}$ and others & $\begin{array}{l}\text { There is increasing evidence that multiple mutations emerge for all PIs and cross resistance } \\
\text { in this class is common. High level resistance requires the accumulation of multiple (3-7) } \\
\text { mutations and as more mutations emerge cross resistance is more readily detected. }\end{array}$ \\
\hline Indinavir & $\mathrm{M} 46 \mathrm{I} / \mathrm{L}, \mathrm{V} 82 \mathrm{~A} / \mathrm{F} / \mathrm{T}$ and others & As above \\
\hline Saquinavir & G48V,L90M, and others & As above \\
\hline Nelfinavir & $\mathrm{D} 30 \mathrm{~N}$ and others & As above \\
\hline Amprenavir & $\mathrm{I} 50 \mathrm{~V}, \mathrm{M} 46 \mathrm{I}, \mathrm{I} 47 \mathrm{~V}$ & Little in vivo data to date \\
\hline
\end{tabular}

*Amino acids: $\mathrm{A}=$ alanine; $\mathrm{C}=$ cysteine; $\mathrm{D}=$ aspartic acid; $\mathrm{E}=$ glutamic acid; $\mathrm{F}=$ phenylalanine; $\mathrm{G}=$ glycine; $\mathrm{H}=$ histidine; $\mathrm{I}=$ isoleucine; $\mathrm{K}=$ lysine; $\mathrm{L}=$ leucine;

$\mathrm{M}=$ methionine; $\mathrm{N}=$ asparagine; $\mathrm{P}=$ proline; $\mathrm{Q}=$ glutamine; $\mathrm{R}=$ arginine; $\mathrm{S}=$ serine; $\mathrm{T}=$ threonine; $\mathrm{V}=\mathrm{valine} ; \mathrm{W}=$ tryptophan; $\mathrm{Y}=$ tyrosine.

current HIV antiretroviral drugs are summarised in table $1 .^{3}$

\section{Monitoring HIV infected individuals}

MEASURING VIRAL LOAD

The efficacy of antiretroviral therapy is monitored by changes in plasma viral load. Ideally, the drug regimen should reduce the viral load to undetectable amounts (typically $<400$ copies $/ \mathrm{ml}$ ) to minimise the risk of viral resistance developing. Early data suggest that an achievement of $<50$ copies $/ \mathrm{ml}$, as determined in more sensitive assays, prolongs the virological durability of drug effect. Virological failure is identified as an increase in viral load from the suppressed level and is often associated with the emergence and selection of drug resistant variants. Other factors, however, such as patient compliance must also be considered when a viral load rebound is identified (see later section).
MEASURING DRUG RESISTANCE

"Phenotyping" involves the in vitro determination of drug susceptibility in a virus culture assay and provides a direct measure of biological resistance. As mentioned above, the susceptibility of a virus isolate is usually expressed as the drug concentration required to inhibit viral replication by $50 \%\left(\mathrm{IC}_{50}\right)$. Phenotypic resistance is measured quantitatively as an x-fold decrease in drug susceptibility (or an $\mathrm{x}$-fold increase in $\left(\mathrm{IC}_{50}\right)$. A standardised method for drug susceptibility testing involves a peripheral blood mononuclear cell (PBMC) culture system. This is slow and labour intensive but remains the "gold standard". More recently, methods have been developed for more rapid throughput using a recombinant assay system. In this case, infectious virus is regenerated by recombination of polymerase chain reaction (PCR) products derived from patients' plasma 
(for instance, protease and/or reverse transcriptase genes) with an HIV-1 plasmid clone deleted for the relevant gene. ${ }^{4}$ Early results suggest a good correlation between these assays (Virco Antivirogram or Virologics) and the PBMC system. Nevertheless, the turnaround time for such assays is at least $2-4$ weeks and they are expensive.

The second method of examining drug resistance is "genotyping". This is the analysis of mutations in the viral genome that might confer phenotypic resistance. In some cases high level resistance (100-1000-fold increase in $\left.\mathrm{IC}_{50}\right)$ is caused by a single point mutation. An example of this is the A to G mutation in codon 184 of the reverse transcriptase gene that changes methionine to valine and generates high level lamivudine resistance. ${ }^{5}$ With many antiretroviral drugs, however, the degree of viral resistance is dependent on the number and combination of genotypic changes. High level phenotypic resistance to zidovudine (AZT) is linked with mutations that change amino acids $41,67,70$, 215 , and 219. Individually and in various combinations these mutations produce virus populations with varying phenotypic resistance (as measured by $\mathrm{IC}_{50}$ ). ${ }^{3}$ There are also instances where resistance mutations for one drug reverse resistance to a second drug. For example, mutations at codons 184 and 181 associated with resistance to lamivudine (3TC) and nevirapine respectively, reverse the zidovudine (AZT) resistance conferred by mutations at amino acids 41 and 215 . $^{\circ}$

Recent research has built up a comprehensive and in depth understanding of genotypic changes associated with resistance to different antiretroviral drugs, either in monotherapy or in combination. Mutations selected for by in vitro passage of the virus in the presence of drug do not invariably reflect the mutations observed in vivo, particularly in the context of combination therapy. In the case of protease inhibitors, resistance associated mutations are acquired sequentially. Although the order in which these mutations accumulate may vary for different drugs, it is likely that a larger array of mutations in response to any one drug will confer cross resistance to other drugs within this class.

The effects of the mutations have been correlated with the phenotypic resistance patterns (table 1 and Schinazi et al ${ }^{3}$ ). Confirmation that specific mutations cause drug resistance depends on the demonstration that HIV viral clones into which these mutations are introduced show reduced drug susceptibility in phenotypic assays. These experiments have also shown that some resistance associated mutations do not, in fact, have an impact on drug susceptibility itself, but rather compensate for the detrimental growth effects of resistance mutations elsewhere. ${ }^{7}$ This understanding is extremely important in the move towards predicting therapeutic failure before it results in increased viral replication and damage to the immune system.

Genotypic assays

Although genotypic assays are an indirect method of determining resistance they have a
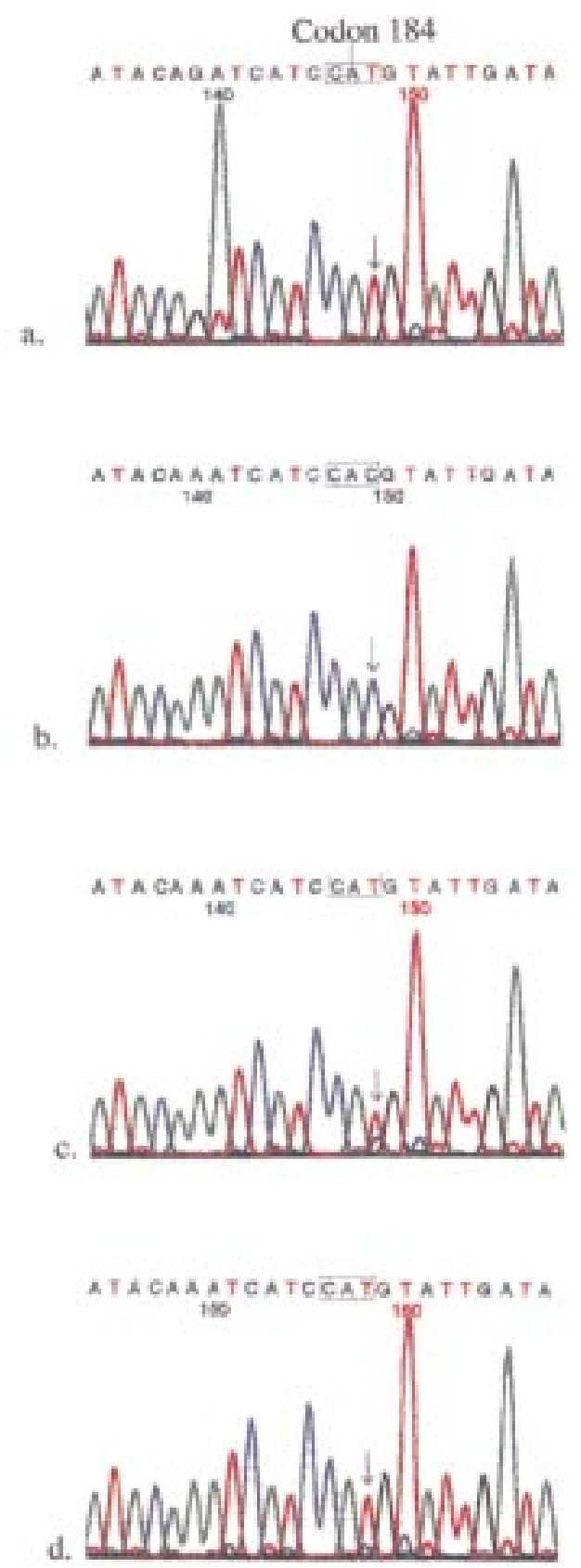

Figure 1 Automated ABI sequence analysis of the codon 184 region of the reverse transcriptase gene of $H I V-1$. The sequence shown is antisense, hence the wild type reads $C A T$ that corresponds to ATG (methionine) in the sense orientation. The mutant sequence reads $C A C$ in antisense corresponding to GTG (valine) in the sense orientation. Patients were tested after treatment failure (identified as $>0.5$ log increase in viral load). Patient (a) was treated with lamivudine and stavudine and patients (b), (c), and (d). were treated with lamivudine and zidovudine. The following sequence types were identified:patient (a) wild type indicating no genotypic resistance to lamivudine; patient (b) mutant type indicating genotypic resistance to lamivudine; patient (c) mixed types indicating partial genotypic resistance to lamivudine; patient (d) wild type indicating no genotypic resistance to lamivudine. (For these patients sequencing of the rest of the reverse transcriptase gene gave concordant results with LiPA, see figure 2.)

major advantage over phenotypic assays in being quick to perform. Genotyping can be completed in 1-2 days whereas phenotyping, dependent on virus isolation and culture, might take up to 8 weeks. At present, the gold standard for detecting point mutations is by nucleotide sequencing of the appropriate viral 


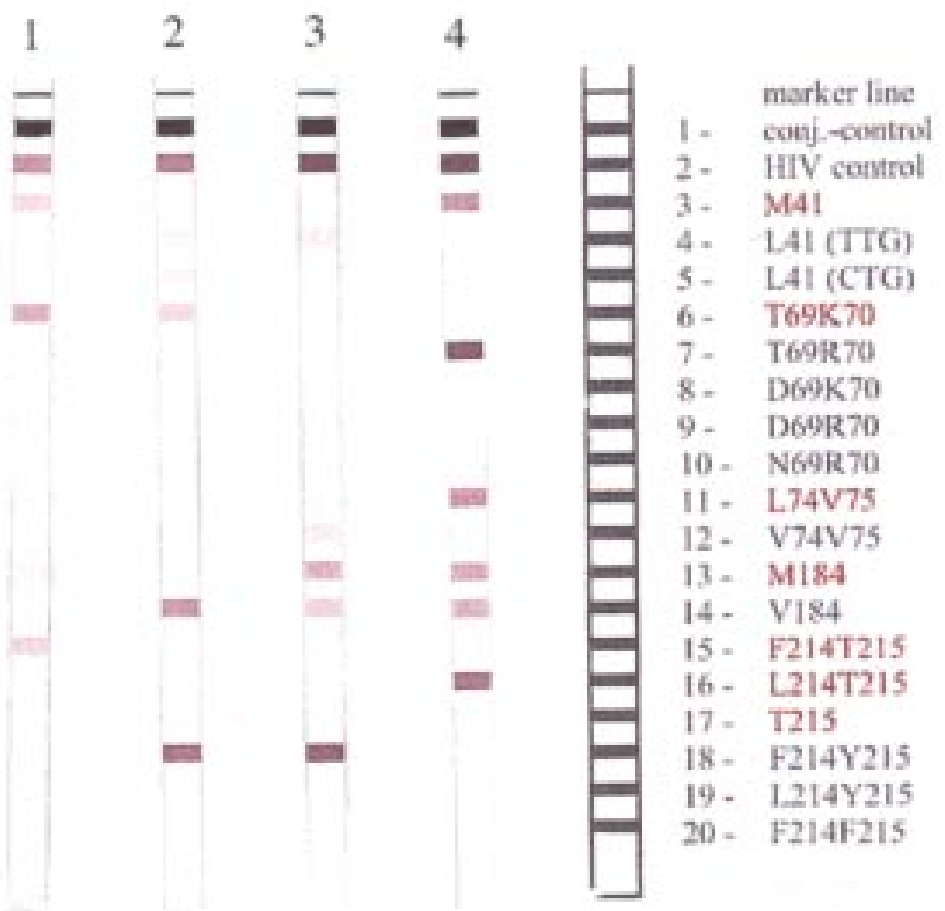

Figure 2 LiPA results for patients failing drug therapy. The patients tested correspond to those described in figure 1 with samples from patients a-d shown in lanes 1-4 respectively. The following sequence types were identified: patient a (lane 1): wild type sequence at all codons tested $(41,70,74,184$, and 215$)$ indicating no genotypic resistance; patient $b$ (lane 2): mixed mutant sequences at codon 41, mutant sequence at codon 215 and also mutant sequence at codon 184, indicating genotypic resistance to both zidovudine and lamivudine; patient c (lane 3): mutant sequence at codons 41, 74 and 215 together with mixed wild and mutant sequences at codon 184 indicating genotypic resistance to zidovudine, didanosine, zalcitabine and partial resistance to lamivudine; patient d (lane 4): mutant sequence at codon 70 and mixed wild and mutants sequences at codon 184 indicating low level genotypic resistance to zidovudine and partial resistance to lamivudine.

genes. To achieve this the viral genome has first to be extracted, usually from plasma, since plasma viral RNA is more representative of actively replicating virus than cellular proviral DNA. The reverse transcriptase and protease genes are then amplified by reverse transcription followed by PCR. The sequence of the DNA fragments produced can be determined by manual sequencing methods or more rapidly by using automated sequencing. Sequencing allows all the point mutations within a particular DNA fragment to be determined simultaneously and usually unequivocally.
Figures $1 \mathrm{a}$ and $\mathrm{b}$ show the detection of wild type and mutant type sequences respectively, at codon 184 of the reverse transcriptase gene by automated sequencing. Problems can arise when there are mixed populations of virus present in a sample. Automated sequencing is able to detect such mixtures when the proportions of each variant are similar as illustrated in figure 1c. When one variant is present as a minor component of the overall population, however, it may be undetectable above background by sequencing (fig 1d) yet still be detectable by other more sensitive techniques (fig 2 (lane 4)). From a clinical viewpoint, the detection of low levels of resistant virus, before complete drug failure might prove vitally important. In order to maintain maximal suppression of viral replication, it might be necessary to alter therapy as soon as a genotypically resistant mutant is identified but the major limitation of genotypic assays is the lack of sensitivity such that minor species may not be readily detected. The advantages and disadvantages of the different techniques discussed in this section are highlighted in table 2 .

Other PCR based techniques have been developed to address this problem of detecting mixed genotypes. The point mutation assay looks at a single nucleotide site rather than the overall sequence. The technique is best described as a solid phase "minisequencing" reaction-that is, the identification of the nucleotide at a single position in the genome. This type of assay has been shown to detect mutant virus at levels as low as $3 \% .^{8} \mathrm{~A}$ radioactive form of this type of assay has been developed as a research tool for the monitoring of AZT combination therapies. ${ }^{8}$ With the recent development of a colorimetric version of the point mutation assay for the detection of mutants of hepatitis B virus ${ }^{9}$ it might be feasible to introduce this type of assay for routine monitoring and epidemiological studies of HIV. Obviously this will be appropriate where a small number of changes lead to resistance; to reveal the complete genotypic pattern of a sample several point mutation assays for different mutational sites must be combined. The main advantages of the colorimetric point

Table 2 Advantages and disadvantages of genotypic assays for HIV drug resistance

\begin{tabular}{ll}
\hline Assay type & Advantages \\
\hline Sequencing (automated) & $\begin{array}{l}\text { Rapid. } \\
\text { Comprehensive information—all nucleotide changes analysed. }\end{array}$ \\
& \\
Genechip system & $\begin{array}{l}\text { Rapid and easy to perform. } \\
\text { Comprehensive information-all nucleotides analysed. } \\
\text { Comparable with ABI sequencing. } \\
\text { Gives information on a large section of the genome in a single } \\
\text { assay run (both RT and polymerase gene analysed } \\
\text { simultaneously). }\end{array}$ \\
Rapid and easy to perform. \\
Line probe assay (LiPA) \\
Semiquantitative. Good at detecting mixed viral populations \\
(sensitivity about 5\%). \\
No specialised equipment required. \\
Rapid and easy to perform.
\end{tabular}

Inexpensive (approximately 10 times cheaper than sequencing). Semiquantitative. Good at detecting mixed viral populations (sensitivity about $5 \%$ ).
Disadvantages

\section{Expensive.}

Specialised equipment required.

Expertise in sequence interpretation needed.

Relatively insensitive for detection of mixed populations (species $<25 \%$ of population not reliably detected).

Expensive.

Specialised equipment required.

Cannot detect previously unknown sequences.

Relatively insensitive for detection of mixed populations (species $<25 \%$ of population not reliably detected)

Very expensive.

Limited number of codons can be analysed. Difficult to interpret individual mutations in absence of other information.

Limited to small number of codons that can be analysed. As for line probe assay difficult to interpret individual mutations in absence of other information. 


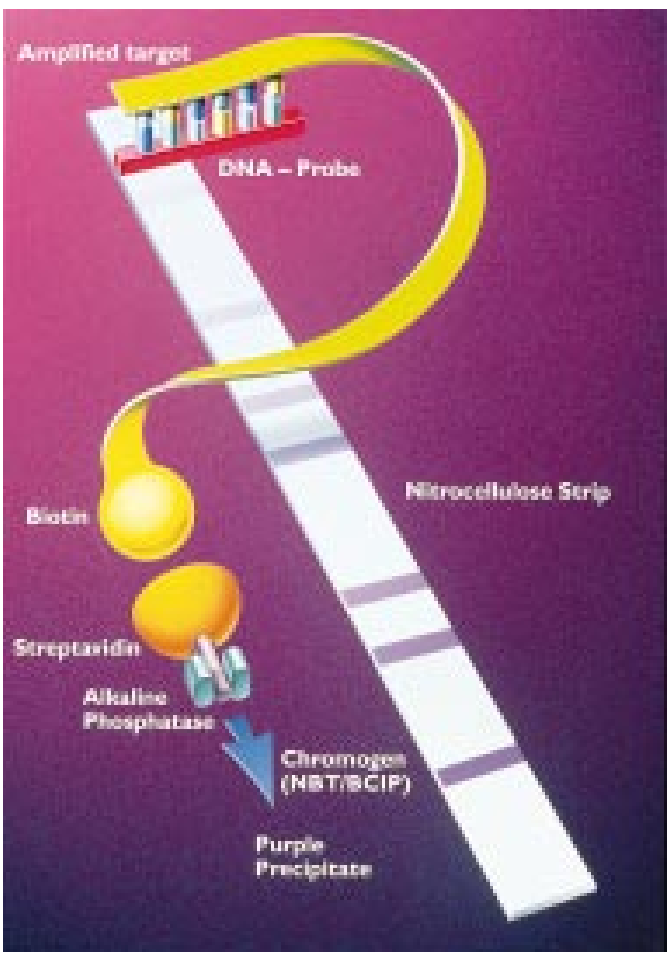

Figure 3 Principle of line probe assay (LiPA) HIV-1 reverse transcription (reproduced with permission form Murex Ltd).

mutation assay are that the reagents are cheap and in the context of a routine diagnostic laboratory no specialised equipment is required (table 2).

At least two commercial genotyping tests are now available including the line probe assay and the Affymetrix GeneChip system. Both tests are based on the fact that single stranded DNA molecules will bind together if the sequences of the molecules are complementary - that is, if there are sufficient base pair matches. This method of nucleic acid hybridisation can be used to analyse a range of mutations with a high degree of specificity and sensitivity.

LIPA HIV-1 RT

The line probe assay (LiPA) detects multiple mutations as follows: the gene of interest is amplified by PCR in such a way that a biotin marker is incorporated into it. The amplified DNA is then added to nitrocellulose assay strips in a hybridisation reaction. The nitrocellulose strips carry a series of DNA oligonucleotide sequences that act as probes that correspond to different wild type and mutant genes. The test DNA will only bind to the strip if the sequences match exactly. By incorporating the biotin marker into the test DNA, a colour reaction can occur allowing the bands where the test sample has hybridised to be visualised (fig 3).

The LiPA shows in one test whether the virus sample contains wild type or mutant nucleotide sequences at various codons $(41,69,70,74$, 184,214 , and 215 ) of the reverse transcriptase gene. ${ }^{10}$ The test is quick and easy to perform and is able to detect mixed populations of viruses. The LiPA results for four patients failing treatment (defined as $>0.5 \log$ rise in viral load) are shown in figure 2 which illustrates the detection of wild type, mutant type, and mixed type sequences at amino acid 184. As mentioned above the LiPA showed mixed wild type and mutant type sequence at codon 184 in one instance (fig 2 (lane 4)) that was not detectable by sequencing (fig 1d). The LiPA thus appears to have a distinct advantage over sequencing in the determination of mixed viral populations (table 2). It should be noted that the sequencing and LiPA results were concordant for all the other codons tested for seven patients (our unpublished data). The main disadvantage of the LiPA is that the number of mutations that can be analysed is limited. Genotypic resistance against NNRTIs and protease inhibitors cannot be detected by the current commercially available version (table 2), although prototype LiPA strips incorporating additional mutation targets are being tested.

\section{THE GENECHIP SYSTEM}

The Affymetrix GeneChip system has been designed to provide accurate sequence information on portions of the HIV genome. ${ }^{11}$ DNA probe sequences are immobilised in an array on a silicon chip in such a way that the length and location of each is known. The sequence of the test sample is then identified by analysis of the hybridisation position within the chip using fluorescence and a confocal microscope. For the HIV polymerase chip there are more than 15000 probe sequences that can detect different strains of HIV-1 with respect to any nucleotide position on the HIV protease gene and first part of the reverse transcriptase gene. Although expensive hardware is required to run the system, it is probably no more costly than automated sequencing and is very user friendly. One disadvantage of this system is that it is unable to detect previously unknown sequences.

\section{Clinical utility of genotypic resistance assays}

What then is the role of HIV resistance assays in clinical practice? They might play a role in the following scenarios.

\section{TO DETERMINE THERAPEUTIC OPTIONS}

FOLLOWING DRUG FAILURE

As more drugs become available, the determinants of second and third line combinations will become more tailored to individual patients, and resistance assays might be useful in this context. The clear demonstration of resistance associated mutations can be used to exclude certain therapies. Conversely, the failure to identify lack of key resistance associated mutations may imply that drug failure is due to poor drug compliance or other pharmacological mechanisms. Indeed, a recent survey of resistance mutations in patients failing triple therapy showed that about $30 \%$ did not have detectable resistance mutations. ${ }^{12}$ On the other hand, the lack of detectable mutations does not exclude their existence at low frequency that might then rapidly emerge in response to a new drug. Recent data suggest that a clinician's suspicion of poor compliance is a strong predictor 
of drug failure-why use an expensive laboratory test to demonstrate the same?

It is evident that interpretation of genotypic data remains complex particularly where patients might have received many different drug combinations over varying periods and with different levels of compliance. In addition, data derived from PI treated patients suggest that such therapy may prime a response to a second PI, which is not necessarily predictable from the majority genomic species at the time of initial PI failure. Ultimately, genotypic assays will only enter routine clinical practice if they are shown to predict virological and clinical responses. Preliminary data supporting this approach have been presented for abacavir where poor virological response to this drug is associated with multiple genotypic changes in RT before initiation. ${ }^{13}$

ASSESSING DRUG SUSCEPTIBILITY IN ANTIRETROVIRAL NAIVE INDIVIDUALS

Transmission of drug resistant viruses has been well documented ${ }^{14}$ and evidence from epidemiological studies and from genotypic data derived from drug naive patients entering clinical trials suggest that the incidence of drug resistance mutations in untreated patients is increasing. ${ }^{15}$ In this respect, pretreatment genotypes might predict the efficacy of antiretroviral treatment. Drug resistance assays might play a role in guiding such therapy in the future.

\section{TO DETERMINE OPTIMAL POST-EXPOSURE} PROPHYLAXIS

Current Department of Health guidelines suggest triple therapy (including a protease inhibitor) for post-exposure prophylaxis following a needlestick injury involving blood from an HIV infected donor. ${ }^{16}$ As an increasing number of HIV patients receive such triple therapy, there is a risk that drug resistant viruses will be transmitted to recipients of such incidents and that post-exposure prophylaxis will be suboptimal. Studies are required to establish the prevalence of resistance within any one setting; if this is significant then perhaps rapid genotyping of source virus will be required to determine optimal post-exposure prophylaxis.

TO DETERMINE OPTIMAL THERAPY IN VERTICALLY INFECTED INFANTS

Few data are available on emergence of resistance in antiretroviral treated pregnant women. None the less, since vertical transmission of drug resistance has been documented it seems reasonable to assess such resistance at delivery.
This could be used to guide subsequent therapy in the infant if necessary.

Genotypic assays that can be used in a routine setting are now available although further development will enhance the amount of information that they can provide. For such assays to make the transition from research tool to routine clinical use, however, additional research is still required. Further evidence that genotyping can be used to predict reliably response to subsequent therapy is needed and also the contribution of genotypic resistance to drug failure must be clarified.

1 Coffin JM. Retroviridae: the viruses and their replication. In BN Fields, DM Knipe, PM Howley, et al, eds. Fields virology. 3rd ed. Philadelphia: Lippincott-Raven, 1996:1767847.

2 Pathak VK, Hu W-S. 'Might as well jump!' Template switching by retroviral reverse transcriptase, defective genome formation and recombination. Sem Virol 1997;8: 141-50.

3 Schinazi RF, Larder BA, Mellors JW. Mutations in retroviral genes associated with drug resistance. Int Antiviral News 1997;5:129-42.

4 Hertogs K, de Bethune MP, Miller V, et al. A rapid method for simultaneous detection of phenotypic resistance to inhibitors of protease and reverse transcriptase in recombinant human immunodeficiency virus type 1 isolates from patients treated with antiretroviral drugs. Antimicrob Agents Chemother 1998;42:269-76.

5 Tisdale M, Kemp SD, Parry NR, et al. Rapid in vitro selection of human immunodeficiency virus type 1 resistant to 3'thiacytidine inhibitors due to a mutation in the YMDD region of reverse transcriptase. Proc Natl Acad Sci USA 1993;90:5653-6.

6 Larder BA, Kemp SD, Harrigan R. Potential mechanism for sustained antiretroviral efficacy of AZT-3TC combination therapy. Science 1995;269:696-9.

7 Ho DD, Toyoshima T, Mo H, et al. Characterisation of human immunodeficiency virus type 1 variants with increased resistance to C2-symmetric protease inhibitor. $\mathcal{f}$ increased resistance to

8 Kay S, Loveday C, Tedder RS. A microtitre format point mutation assay: application to the detection of drug resistance in human immunodeficiency virus type 1 infected patients treated with zidovudine. F Med Virol 1992;37:241-

9 Ballard A, Boxall EH. Colourimetric point mutation assay for detection of pre-core mutants of hepatitis B. F Virol Methods 1997;67:143-52.

10 Stuyver L, Wyseur A, Rombout A, et al. Line probe assay for rapid detection of drug-selected mutations in the human immunodeficiency virus type 1 reverse transcriptase gene. Ammunodeficiency virus type 1 reverse transc

11 Kozal MJ, Shah N, Shen N, et al. Extensive polymorphisms observed in HIV-1 clade B protease gene using highdensity oligonucleotide arrays. Nat Med 1996;2:753-9.

12 Mayers DL, Gallahan DL, Martin GJ, et al. Drug resistan genotypes form plasma virus of HIV-infected patients failing combination drug therapy. International Workshop on HIV Drug Resistance, Treatment Strategies and Eradication, St Petersberg, FL. June 1997 (abstract 80).

13 Lanier ER, Stone C, Griffin P, et al. Phenotypic sensitivity to 1592 (Abacavir) in the presence of multiple genotypic
mutations: correlation with viral load response. 5th Conference on Retroviruses and Opportunistic Infections, Chicago, 1998 (abstract 686)

14 Imrie A, Beveridge A, Genn W, et al. Transmission of human immunodeficiency virus type 1 resistant to nevirapine and immunodeficiency virus type 1 resistant to

15 Rubio A, Leal M, Pineda JA, et al. Increase in the frequency of mutation at codon 215 associated with zidovudine resistance in HIV-1-infected antiviral-naive patients form 1989-1996. AIDS 1997;11:1184-6.

16 Department of Health. Guidelines for HIV post-exposure prophylaxis. London: Stationery Office, 1997. 